\title{
ACTORES Y PROCESOS PATRIMONIALES: LA EXPERIENCIA PUEBLANA
}

\author{
PATRIMONIAL'S ACTORS AND PROCESS: THE PUEBLA \\ EXPERIENCE
}

Enviado em 04 de setembro de 2015

Aceito em 20 de novembro de 2015

María del Carmen Fernández de Lara Aguilar ${ }^{1}$

Alejandro Enrique Benítez Barranco ${ }^{2}$

\begin{abstract}
Resumen: De los innumerables problemas a los que se enfrenta la conservación del patrimonio edificado hoy día además de las guerras, es sin duda, las que tiene que ver con siniestros de carácter natural. Revisando la historia de las contingencias que han afectado a la República Mexicana, y más concretamente al Estado de Puebla, en los últimos cincuenta años; se identifican las lluvias, y los movimientos telúricos como los fenómenos naturales más recurrentes que han vulnerado no solo el patrimonio material, sino también el inmaterial, el cual al estar presente en eventos festivos y tradiciones, trastoca la vida social de las comunidades afectadas, y sin duda, atenta contra los elementos de identidad, que contribuyen a la diversidad cultural del país. Bajo un escenario poco alentador al momento del suceso natural, la participación social cobra relevancia; y el reconocimiento que la propia comunidad hace de los valores intangibles y tangibles de los bienes materiales dañados, es lo que lleva a decidir acciones de intervención casi de forma inmediata, siendo incluso un ejemplo de gestión patrimonial sorprendente por el compromiso constante de las comunidades con dicho patrimonio.
\end{abstract}

Palabras clave: Afectación del patrimonio. Catástrofes naturales. Puebla, México.

Abstract: Among the innumerable problems confronted currently in the area of architectural heritage conservation, in addition to armed conflicts, natural disasters figure prominently. Looking back at the history of disasters that have affected the Republic of Mexico, and more specifically the state of Puebla, in the past fifty years, rain and earthquakes are the most frequent natural phenomena

\footnotetext{
1 Arquiteta, professora da Faculdade de Arquitetura da Benemerita Universidad Autonoma de Puebla. Trabalha com patrimônio cultural, conservação e restauro. E-mail: carmina_fdezdelara@yahoo.com.mx

2 Arquiteto, professor da Faculdade de Arquitetura da Benemerita Universidad Autonoma de Puebla. Trabalha com patrimônio cultural, conservação e restauro. E-mail: aenrique2000@yahoo.com.mx
} 
that have damaged both material and immaterial cultural heritage. These natural disasters have also affected festive events and traditions, and the social lives of communities, threatening elements of identity that contribute to the cultural diversity of the nation. Although the panorama is disheartening in the moment of an occurrence of a natural disaster, it is precisely at that crucial time when societal participation becomes relevant. The community's recognition of the damages to their material and immaterial cultural heritage is what leads to their almost immediate intervention in the crisis. This reaction is a model of heritage management that is surprising for the constant commitment demonstrated by the communities to their cultural heritage.

Keywords: Damage to cultural heritage. Natural disasters. Puebla, Mexico.

\section{INTRODUCCIÓN}

La entidad poblana cuenta con 217 municipios, en ellos un importante patrimonio civil y religioso de los siglos XVI al XX, se ha erigido en el devenir del tiempo, su conservación por Ley, se asume, la realice el Instituto Nacional de Antropología e Historia, instancia oficial de carácter federal, quien en realidad es solo una instancia coadyuvante en la protección y restauración de los bienes muebles e inmuebles a conservar en el territorio nacional. Ante el universo de patrimonio cultural que existe en el país, y bajo la Ley Federal sobre Monumentos y Zonas Arqueológicos, Artísticos e Históricos, este Instituto, junto con autoridades estatales, municipales, asociaciones civiles y la población en general, se convierten en los encargados de asumir el compromiso social de conservar el patrimonio.

En pequeñas localidades del territorio poblano, se ignora la existencia de esta Ley, y es la propia población quién ha asumido las acciones de conservación y protección, principalmente de inmuebles religiosos, pero no por su valor histórico, arquitectónico o artístico, sino por ser el espacio que por generaciones ha contribuido a la cohesión social, ya que en él, se deciden las festividades del pueblo, en él se determinan las fiestas paganas, las celebraciones familiares como bodas, bautizos, primeras comuniones individuales y comunitarias que allí se desarrollan, en él se da también el último adiós a los seres queridos cuando fallecen; es así, que la vida diaria transcurre en torno al edificio religioso. Esta particularidad, transforma el sentido de las obras materiales patrimoniales, a partir de aspectos inmateriales, los cuales generan un fuerte sentimiento de pertenencia dentro de los miembros de la comunidad.

El trabajo que se presenta, es un ejemplo de ese otro significado del patrimonio edificado, no del que se define en reuniones de especialistas, o en documentos 
nacionales o internacionales, sino el que los propios usuarios le dan, el que día a día está presente, y el que, se convierte en eje de las decisiones sobre su conservación; decisiones que se acrecientan en momentos de siniestros, tal como se dará a conocer; donde se muestra en todo momento, que el bien inmueble es un producto cultural que pertenece a toda la comunidad, y ella es la responsable de su conservación.

\section{SAN SALVADOR CHACHAPA, PUEBLA. CASO DE ESTUDIO}

El caso que interesa destacar es el de la comunidad de San Salvador Chachapa, localidad perteneciente al Municipio de Amozoc de Mota, ubicada a 15 kilómetros al oriente de la ciudad de Puebla (capital del Estado de Puebla, entidad de la República Mexicana). Cuenta con una población de cerca de 20 mil habitantes. La religión que profesa la mayor parte de la población es la católica, y una de las construcciones más representativa del sitio, por obvias razones, lo es la Parroquia, iglesia que administra los sacramentos, y controla un territorio donde varias capillas están bajo su jurisdicción, siendo ella la cabecera. La advocación de la iglesia es el Divino Salvador, representada en la imagen del 'misterio de la transfiguración de Cristo'. Las festividades civiles y religiosas se relacionan con el santo patrono de la localidad, de ahí la importancia del inmueble que resguarda la imagen de la figura 2.

Figura 1. Mapa de México en el que se localiza el Estado de Puebla y el Municipio de San Salvador, Chachapa.

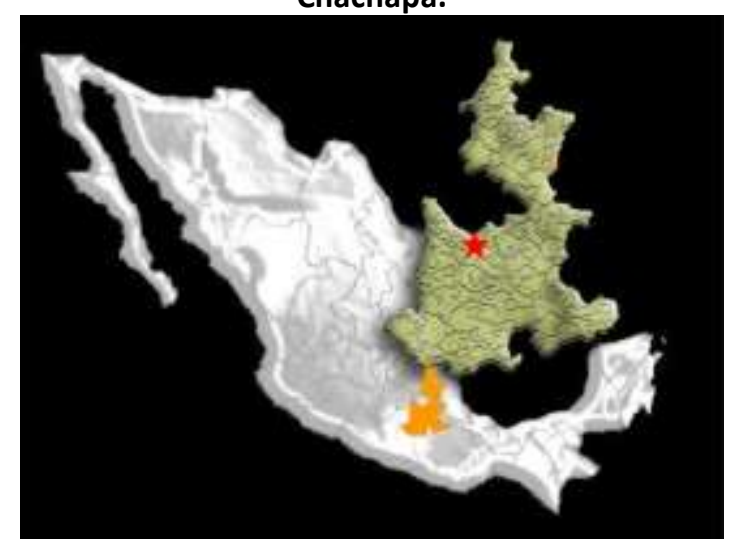

Los movimientos sísmicos en la región son comunes, y los de mayor intensidad, se habían consignado según fuentes oficiales de la siguiente manera: "el período de retorno medio de temblores de magnitud mayor a 6.5 en la zona es de 20 a 30 años"3, aunque en los últimos diez años, estos se han presentado con más frecuencia. En 1999 un sismo de intensidad 6,7 en la escala de Richter afectó siete Estados del centro y oriente de la República Mexicana, el Estado de Puebla entre ellos. El Servicio Sismológico

\footnotetext{
${ }^{3}$ Sistema Nacional de Protección Civil. "El sismo de Tehuacán del 15 de junio de 1999", p. 9.
} 
Nacional (SSN) proporcionó las siguientes coordenadas focales: latitud 18.20 norte, y longitud 97,470 oriente, con una profundidad del sismo de 60 u 80 kilómetros. Su epicentro se estableció a 20 kilómetros aproximadamente de la ciudad de Tehuacán en el Estado de Puebla ${ }^{4}$, y a 55 kilómetros al noreste de la ciudad de Huajuapan de León en el estado de Oaxaca.

La intensidad del fenómeno natural y lo atípico de su comportamiento, dejó 1800 monumentos históricos civiles y religiosos de los Estados de México, Morelos, Tlaxcala, Puebla, Veracruz, Guerrero y Oaxaca con daños de diversas consideraciones en su estructura. A estos inmuebles, se debe sumar daños en caminos, puentes, infraestructura educativa, recreativa, de salud, viviendas y algunos edificios contemporáneos. A pesar de la intensidad de sismo, las pérdidas humanas fueron pocas, no así, la pérdida de inmuebles patrimoniales, la cual se hacía evidente.

En el caso del Estado de Puebla en particular, los edificios históricos dañados llegaron a 789, en cien de los 217 municipios que conforman el territorio poblano. En la ciudad capital, los daños abarcaban innumerables inmuebles ligados a la vida religiosa, económica, política, social y cultural, presentaban daños 59 edificios religiosos e innumerables edificios civiles de los 2,619 que componen la zona de monumentos históricos; área incluida en la Lista indicativa de la UNESCO por su valor excepcional y universal. El caos, el temor producto de las réplicas, y el número de inmuebles dañados, hacía difícil iniciar con acciones de reconocimiento, y mucho más con intervenciones; sin embargo, los trabajos debían empezar, y como siempre sucede en estos casos, los trabajos empiezan por la ciudad capital, y las ciudades de mayor importancia, dejando para un segundo momento las localidades más pequeñas o más alejadas de la capital.

Figura 2. Daños provocados al patrimonio por el efecto telúrico

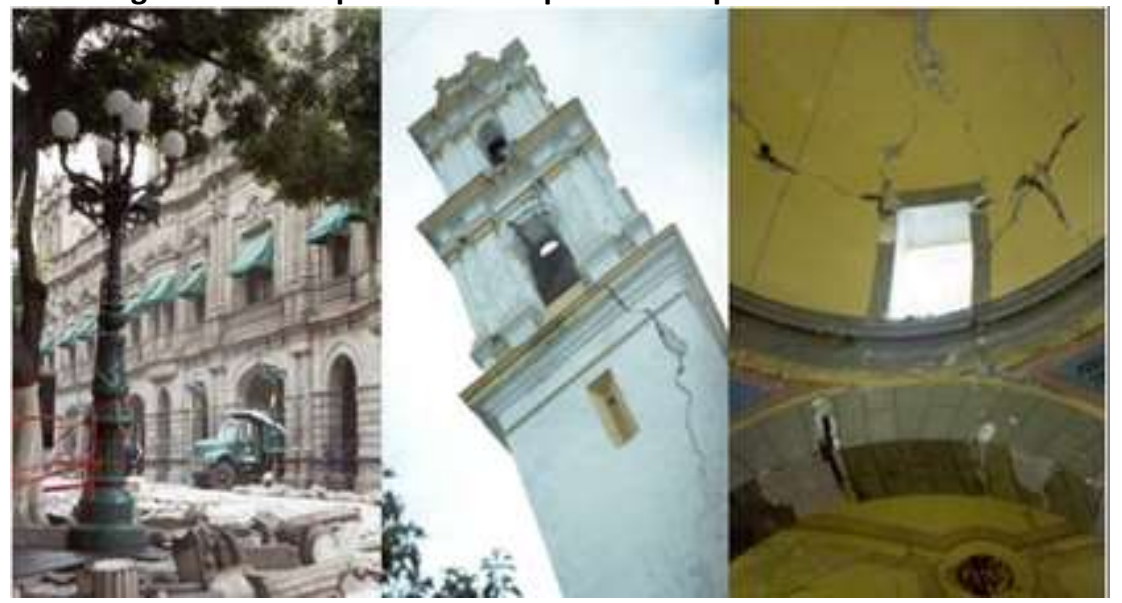

Fuente: Fotografía de A. E. Benítez Barranco - junio 1999.

\footnotetext{
${ }^{4}$ De ahí que las personas refieren el movimiento telúrico como el sismo de Tehuacán.
} 
Solo el ejército mexicano organizado en la Fuerza de Apoyo en Casos de Desastres, intervino en todos los lugares donde hubo afectación, a partir del Plan DN III $E$, que conlleva acciones protocolizadas de búsqueda y rescate de personas, evacuación de comunidades en riesgo, administración de albergues, información permanente a la población, protección de las familias, además de seguridad y vigilancia de las áreas afectadas. Sin duda un acierto en momentos de gran vulnerabilidad de la población. Las acciones en el patrimonio edificado en todo el territorio poblano quedaron para un segundo momento por obvias razones.

Determinar y cuantificar los daños que produjo el evento natural no resultaba fácil, aún cuando se evidenciaban muchos de ellos; el desconocer las condiciones estructurales y de conservación en las que se encontraban los inmuebles antes del siniestro, para definir lo que el evento natural les había ocasionado, resultó una labor titánica. En el caso de la Parroquia de San Salvador, inmueble propiedad federal por ley, para ser atendido, fue necesario establecer primero que de acuerdo al artículo 33 de la Ley Federal sobre Monumentos y Zonas Arqueológicos, Artísticos e Históricos era un monumento artístico, por la fecha de construcción, que se trataba de un bien inmueble que reviste un valor estético relevante por atender características de representatividad, y por estar inserto en una corriente estilística determinada; pero al contener anexos de etapas anteriores al siglo XX, parte del inmueble debía ser considerado como monumento histórico. Solo a partir de esta precisión podría recibir la atención y el apoyo federal para su restauración.

Se realizó el análisis fino y detallado de los daños que el sismo le produjo al inmueble, considerando la medición de la intensidad sísmica, la magnitud del temblor, las condiciones del subsuelo, las características de los materiales y sistemas constructivos empleados en la fabricación, lo que permitió contar con el diagnóstico de los problemas que presentaba el templo.

De manera general, podemos señalar las siguientes afectaciones: desprendimientos de aplanados al interior y exterior del inmueble sin riesgo aparente; pérdida de molduras al interior y exterior; grietas en las dos torres del templo con riesgos de colapso (ver figura 2); pérdida de remate en torre izquierda por colapso (cupulin); desplome de claves de arcos en ambas torres con posibilidades de algún tipo de contingencia (ver figura 3), y grietas en bóveda de la nave principal sin peligro aparente. Mención especial son los daños que presentaba la barda atrial, la cual se colapso en su totalidad, solo la portada principal se mantuvo en pie, presentando innumerables grietas (ver figura 4). También se produjo la pérdida de la verticalidad de la portada (ver figura 5), situación que ponía en riesgo esta parte del bien patrimonial, e incluso había riesgo para la población al transitar por el sitio. Ante estos daños, el inmueble no pudo seguir 
siendo usado, a pesar de ello, las celebraciones se hicieron al aire libre y a un costado del templo, para vincularse con él, de alguna manera.

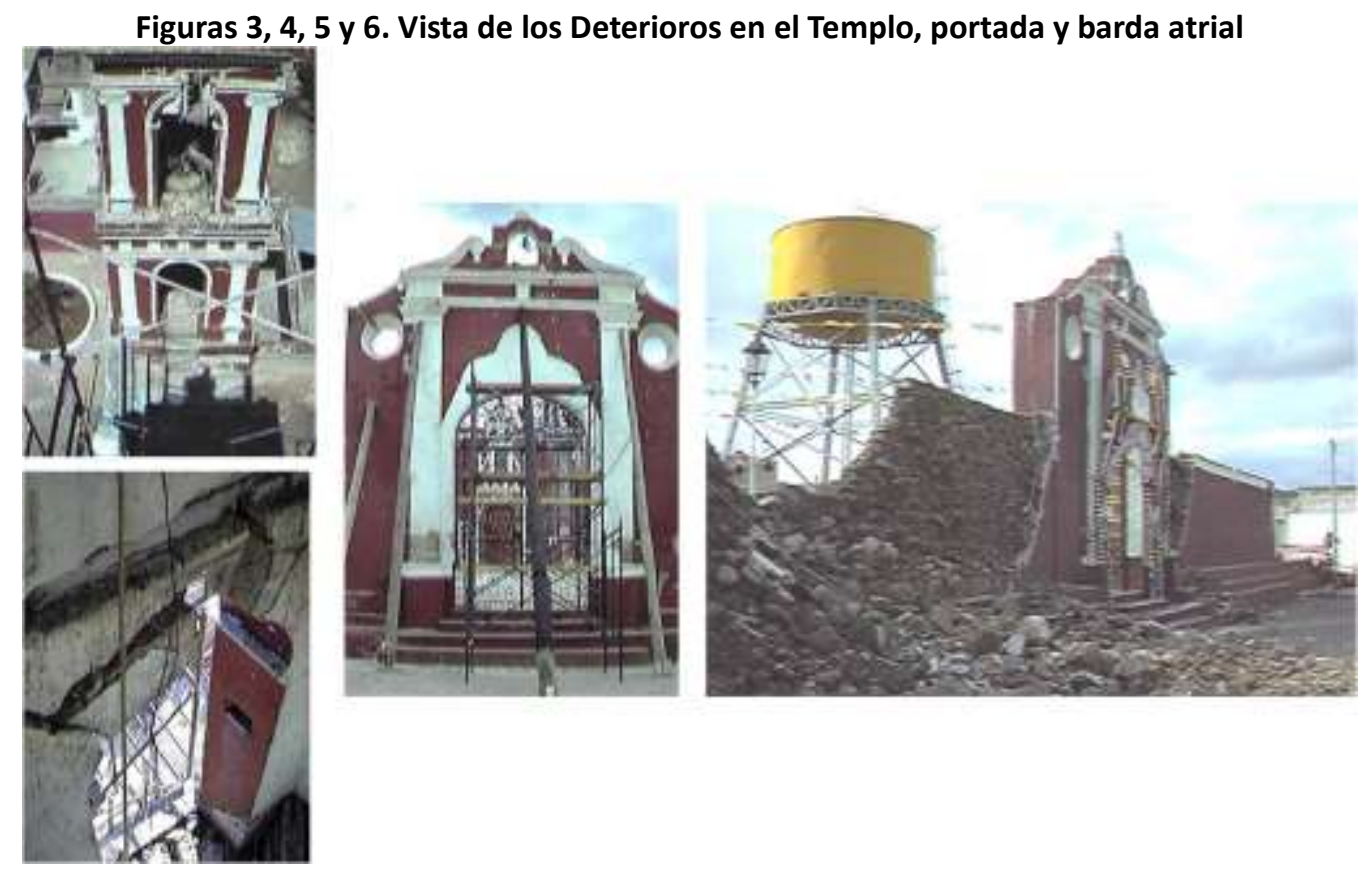

Fuente: A. Enrique Benítez Barranco

Cabe señalar que otros edificios como la presidencia municipal, escuelas y viviendas, presentaban daños producto del fenómeno natural, sin embargo, la comunidad concentró toda su atención en el templo, y el resto de los inmuebles se intervinieron posteriormente.

Pasada la contingencia, y aún en espera de réplicas, la autoridad gubernamental de los tres niveles de gobierno a través de las dependencias relacionadas con la cultura y el patrimonio, establecieron el Comité Técnico Estatal, a partir del Instituto Nacional de Antropología e Historia, y la Secretaria de Cultura entre otras dependencias; iniciando así la cuantificación y valoración de los inmuebles afectados en todo el Estado; hecho que permitiría asignar recursos federales para atender los trabajos de restauración del patrimonio edificado. El vacío de normatividad sobre la forma de atender estos casos, hizo que se actuara con mucha improvisación y tiempo para lograr tener un primer diagnóstico y definir las acciones conducentes a seguir para cada inmueble de las localidades. Las acciones se concentraron en la capital del Estado, ciudades principales y de mayor población, dejando en la indefensión a pequeñas poblaciones, aún cuando la gravedad de las afectaciones en el patrimonio edificado fuera alarmante

La Parroquia de Divino Redentor en la localidad de San Salvador Chachapa, no estaba dentro de las prioridades, a pesar de su cercanía con la ciudad capital. Este hecho, y el no uso del inmueble religioso, fue lo que originó que los propios habitantes junto a las autoridades civiles y religiosas, asumieran la responsabilidad de atender este 
patrimonio edificado. Es así que la comunidad a través de una amplia y real participación social, decide definir acciones que permitieran atender los efectos destructores del movimiento telúrico. Sin la presencia de autoridades de carácter federal o estatal, y sin esperar la ayuda financiera asignada, crean el Comité para la reconstrucción de la Parroquia del Divino Salvador, integrado por autoridades administrativas (Presidente Auxiliar), eclesiásticas (el sacerdote), el comisariado ejidal, líderes sociales (Comisariado Ejidal), los ocho mayordomos 5 , y algunos ciudadanos que la propia comunidad nombró.

El Comité se transforma en una estructura social homogénea con funciones definidas, a partir de las necesidades que se irían presentando; las acciones que se tenían que llevar a cabo en el bien inmueble, se fueron definiendo con la asesoría de un especialista en estructuras históricas y restauración, y un equipo calificado en restauración. Las sesiones en un principio fueron permanentes, y como se fue avanzando y resolviendo problemas, se espaciaron a cada semana, lo que permitió un avance significativo en la habilitación del templo.

A continuación se presenta un esquema, de manera gráfica, para entender cómo y para qué se organiza la comunidad.

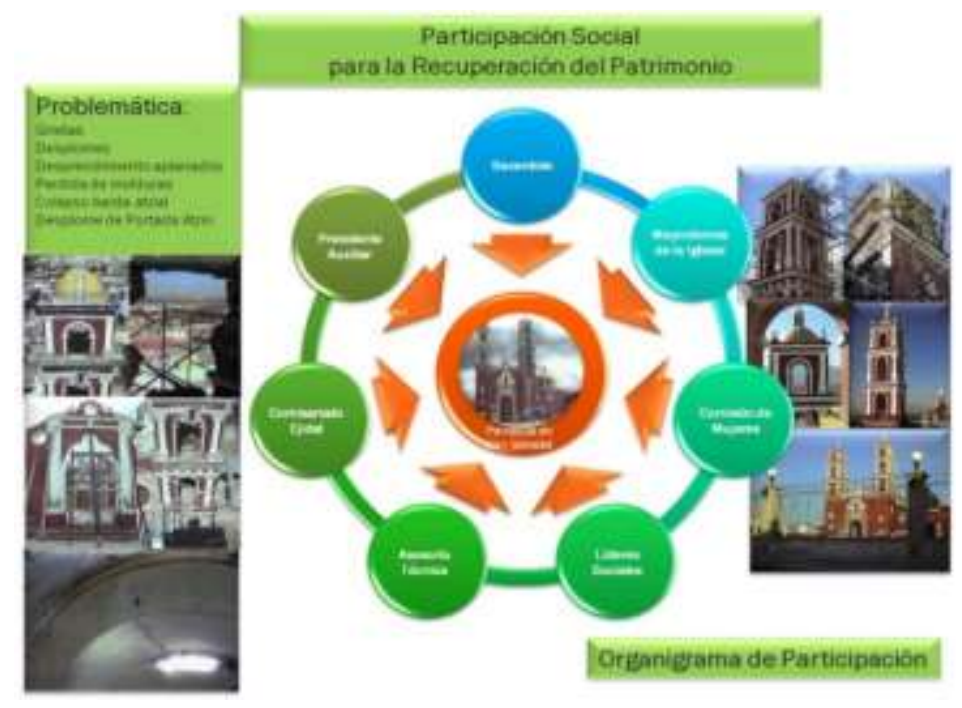

A partir de un proyecto socialmente compartido, al interior del Comité se definieron cargos y comisiones para atender las necesidades que se presentaban, siendo la más importante, los recursos financieros. La población femenina a través de cooperación semanal casa por casa, elaboración de alimentos para venta, organización de eventos sociales, culturales y recreativos, fueron capaces de reunir los recursos que de manera semanal se requerían para solventar los trabajos. Hubo también aportaciones

\footnotetext{
${ }^{5}$ Miembros de la comunidad, con cargo de autoridad que se ocupan de la organización de las actividades religiosas, el mantenimiento del templo, las fiestas patronales, entre otras tareas.
} 
personales de patrocinadores; y la responsabilidad de la administración de los recursos financieros por unanimidad, recayó en el sacerdote de la Parroquia (Pbro. Ignacio Muñoz Cortés).

Entre las acciones que se llevaron a cabo, después del dictamen de daños, y elaboración del proyecto de intervención, fue el aseguramiento del inmueble a través de apuntalamientos, el retiro de escombro producto de colapso, inyección de grietas en bóvedas y torres, atención a fisuras, reestructuración de la torre mediante pernos y placas de acero, integración de aplanado, integración de molduras y pinturas; siempre respetando los valores intrínsecos del monumento, y en apego a las especificaciones que establece el Instituto Nacional de Antropología e Historia. En estricto apego a la normatividad vigente, el Comité decidió llevar a cabo la gestión para el trámite de la licencia para intervenir el inmueble religioso, lo que sin duda pone en alto, el compromiso real que asumieron los pobladores.

Cabe señalar que el inmueble estuvo rehabilitado, mucho antes que el resto de los bienes patrimoniales afectados y que fueron intervenidos a través del Programa de Reconstrucción del Gobierno Federal, donde la participación de constructoras, contratistas, arquitectos e ingenieros, garantizaría menor tiempo y mejores resultados a pesar de que estos trabajos de restauración contaron con recursos financieros autorizados a través del Fondo Nacional de Desastres (FONDEN) ${ }^{6}$, publicado en el Diario Oficial de la Federación el 29 de Febrero del 2000; y administrados a través del Comité Técnico Estatal para la Preservación del Patrimonio Histórico (CTEPPH), integrado éste, por el Secretario de Cultura, el Director del Instituto Nacional de Antropología e Historia, representantes del Consejo Nacional para la Cultura y las Artes, la Secretaria de Gobernación y otras dependencias del Gobierno del Estado de Puebla, así como los presidentes municipales. La diversidad y amplitud de este Comité, y el número de inmuebles que se venían interviniendo simultáneamente, en ocasiones no permitió los resultados esperados.

Regresando al tema del presente estudio de caso, es posible establecer que el éxito de la experiencia, se sustenta en la interacción que existe entre la sociedad y su producción material (la parroquia), lo anterior implica la participación de todos los actores de la misma, vinculados al patrimonio en cuestión, a partir de las relaciones inmateriales que existen en torno a él. La forma de organización espontánea y natural para poner nuevamente en uso el edificio religioso, y el reconocimiento del templo como el espacio social más representativo de la localidad, sin duda fue determinante para los

${ }^{6}$ Se autoriza a la Secretaria de Hacienda y Crédito Público vía el FONDEN, a otorgar recursos que permitan
atender a la población damnificada, los daños sufridos en la infraestructura pública, bosques, áreas
naturales protegidas y monumentos arqueológicos, artísticos e históricos con motivo de un desastre natural. 
resultados, además, la pluralidad de los miembros que formaron parte del Comité, y la igualdad en la responsabilidad, fue otro acierto.

Otro aspecto importante a destacar en esta estrategia, es la participación de dos de los actores civiles y religiosos que jugaron un papel determinante en la rehabilitación de la Parroquia, los mismos son: los ocho mayordomos y la comisión de mujeres. La mayordomía es una tradición en las actividades religiosas del lugar, son las personas que asumen públicamente la responsabilidad de atender todo lo relacionado con la fiesta patronal y demás festividades religiosas. Se responsabilizan desde de las mañanitas al Santo Patrono, la organización y realización de las procesiones por las calles de la población, las misas, el adorno floral de la iglesia, los juegos pirotécnicos, y el día de la festividad principal, hasta la comida para todos los visitantes, por el tiempo que dure la celebración. Todas estas actividades son realizadas por estos personajes en un lapso de un año, su investidura tiene implicaciones, políticas, sociales y culturales, de ahí que los mayordomos, sean personajes ampliamente reconocidos y respetados por la comunidad.

Otro de los actores sin duda, fueron las mujeres, su participación sean estas madres, esposas, hermanas e hijas que tradicionalmente tienen un papel subordinado a las mayordomías, en este caso en particular, asumieron un total liderazgo en las reuniones, en la toma de decisiones, en la colecta casa por casa, y en la organización de todo tipo de eventos semana a semana. En ellas recayó el peso de la recolección de las diferentes contribuciones económicas que se dieron, su trabajo fue permanente, y nunca estuvo subordinado a sus actividades familiares diarias, demostraron disposición absoluta a participar, en todas las labores que el Comité para la Reconstrucción determinó. El compromiso asumido concluyó, al momento en que se llevó a cabo la primera celebración eucarística en el inmueble religioso, la cual estuvo acompañada de una gran festividad, que puso de manifiesto la preservación de la herencia material, y el fortalecimiento de la identidad.

No es posible afirmar que todas las actividades desarrolladas para la recuperación del bien patrimonial, fueron satisfactorias y sin problemas; la solución a la falta de fondos económicos, era lo que siempre estaba en la mesa de la discusión; pero la rendición de cuentas, y la transparencia en el uso de los recursos monetarios y materiales a través de informes semanarios, expuestos al final de las ceremonias religiosas, y exhibidos incluso en periódicos murales, garantizó que los habitantes de San Salvador Chachapa supieran que se hacía con los recursos que aportaban.

Otro escenario de esta misma experiencia lo fue el proyecto de intervención y la obra a restaurar, el primero estuvo expuesta a la mirada de los habitantes, la población, conocían el proyecto de intervención, el cual se expuso primero al Comité de 
Reconstrucción de la Parroquia, y después se presentó a la población en general; de ahí que los reportes de avances en los trabajos de restauración y rehabilitación del monumento, siempre fueron constatados con la obra misma.

La estrategia resultó eficaz, la capacidad organizativa y operativa del Comité, rebasó las acciones de rehabilitación de las propias instancias oficiales. En casos de desastres naturales la recuperación del patrimonio no resulta tarea fácil, pero cuando la población en su conjunto decide participar y colaborar en la intervención de los inmuebles afectados, el verdadero sentido social del patrimonio se hace presente.

\section{CONCLUSIONES}

La descripción de la experiencia por si misma resulta importante, pero más importante hubiera sido que el siniestro telúrico de junio y su consecuente de septiembre de 1999 dejaran establecidas acciones protocolizadas de organización, gestión e intervención en los edificios patrimoniales afectados por siniestros de tipo natural como el ocurrido. Se observó como la apropiación colectiva de la conservación siempre rendirá más frutos, que la suma de esfuerzo concertados de las diferentes instancias de gobierno. Este trabajo es un claro ejemplo.

Las diversas formas de participación que asumieron las comunidades en algunas localidades y ciudades de la entidad poblana, cuando sus bienes culturales religiosos se vieron amenazados, debió ser aprovechada pasada la contingencia con el fin de poder atender el innumerable patrimonio que se vio dañado. Así mismo, se debe reconocer que para los habitantes de esta localidad, la restauración de la Parroquia del Divino Salvador, no corresponde a la recuperación del patrimonio edificado, sino al espacio social, donde un conjunto de formas de cultura tradicional y popular está presente, y que la cohesión social que el espacio y las actividades que en él se llevan a cabo produce, están por encima de cualquier dañado o afectación de la naturaleza.

No debe confundirse la recuperación de este bien cultural material, como el rescate de un bien patrimonial más, sino como la recuperación de una manifestación material de la cultura, en donde lo intangible se transforma en tangible, y que sumado a la obra material, hace que la restauración tenga otro sentido.

El respeto y los valores que tiene el inmueble para la comunidad, no se le asigna, se va adquiriendo con el uso que cada miembro de la población hace del espacio, cuando los hijos contraen matrimonio, cuando se bautiza al familiar, cuando se presentan los niños a los 3 años, cuando se hace la primera comunión, cuando se termina una etapa escolar, y tantas y tantas otras celebraciones más, de las que el templo es testigo y parte importante en la vida de cada habitante de la comunidad. La experiencia permitió a 
todos los interesados en el patrimonio, verlo con sensibilidad y mayor amplitud, evitando limitar la intervención a solo el daño físico.

Resumiendo, si se consideran los valores que los inmuebles religiosos o no tienen para quién lo usa o habita, todos nos volveríamos responsables de su conservación, y con la existencia de protocolos de actuación; sin duda habrá daños materiales en caso de siniestros, pero la intervención expedita y puntual, tenderá a que las pérdidas en el patrimonio material e inmaterial sean menores.

\section{REFERENCIAS}

CONACULTA/INAH. Memorias FONDEN: rehabilitación de inmuebles históricos dañados por los sismos de junio y septiembre de 1999 en los estados de Guerrero, Estado de México, Morelos, Oaxaca, Puebla, Tlaxcala y Veracruz. México: Consejo Nacional para la Cultura y las Artes, 2000.

H. AYUNTAMIENTO de Puebla. Memorias de un sismo. México: Dirección General de Obras Públicas, 2002.

INAH. Ley Federal sobre monumentos y zonas arqueológicos, artísticos e históricos. México: Instituto Nacional de Antropología e Historia, 1995.

INAH. Programa de prevención de desastres en materia del patrimonio cultural. México: Instituto Nacional de Antropología e Historia, 2007.

SISTEMA Nacional de Protección Civil. El sismo de Tehuacán del 15 de junio de 1999. México: Ingeniería Estructural y Geotecnia, 2000.

VILADEVALL I GUASCH, Mireia (Org). Ciudad, patrimonio y gestión. México: Benemérita Universidad Autónoma de Puebla. Gobierno del Estado de Puebla, 2001. 